\title{
Looking for Very Low-Mass Pre-Main Sequence Objects with SDSS
}

\author{
Peregrine M. McGehee \\ MS H820, Los Alamos National Laboratory, Los Alamos, NM 87545 \\ Suzanne L. Hawley \& Kevin R. Covey \\ Department of Astronomy, Box 351580, University of Washington, \\ Seattle, WA 98195
}

\begin{abstract}
Determining the process(es) by which brown dwarfs form is key to understanding their intrinsic nature. If their origins are within circumstellar disks they are akin to giant planets. If, on the other hand, they coalesce from molecular cloud cores, then they share a common lineage with low mass stars. These two mechanisms can be distinguished by investigation of young $(<10 \mathrm{Myr})$ substellar objects. If brown dwarfs are small failed stars, we expect to find very low mass analogs of the Classical $\mathrm{T}$ Tauris, with primordial magnetic fields from the molecular cloud collapse. Accretion onto these objects leads to characteristic magnetic activity signatures such as chromospheric and coronal emission resulting in an ultraviolet excess continuum.

The Orion OB1b association (m-M $=7.9,2 \mathrm{Myr}$ ) provides a laboratory for following the strength and occurrence of the accretion process as a function of mass. Studies of the substellar mass function within the sigma Orionis cluster at the southern end of the association indicate that brown dwarfs are common. Based on model isochrones and the SDSS M dwarf sequence we expect the $95 \%$ completeness limit of the "Orion" scans to correspond to 0.1 and 0.03 solar masses for the $u$ and $g$ bands.
\end{abstract}

\section{SDSS Ultraviolet Excess Survey}

Comparison of near-IR and ultraviolet excess techniques shows that as the masses decrease, a higher percentage of young objects are found that exhibit an ultraviolet excess only (Rebull et al. 2002). This is primarily attributed to an increasing contrast between the stellar photosphere and the accretion shock, coupled with a decrease in the contrast between the photosphere and the inner circumstellar disk in less massive, cooler stars.

Magnetospheric accretion can be distinguished from purely chromospheric activity on the grounds of the de-reddened Johnson-Cousins U-V excess exceeding 0.5 magnitudes, corresponding to $\Delta u^{*}<-0.67$.

Comparison of 1 to $10 \mathrm{Myr}$ model isochrones (Baraffe et al. 1998) transformed into the SDSS passbands (York et al. 2000, Smith et al. 2002) indicate that the SDSS should be able to detect lightly extincted objects at and just 
below the Hydrogen burning limit within the Orion OB1b association provided they exhibit a $u^{*}$ excess stronger than that expected for chromospheric activity, i.e., that they are Classical T Tauri analogs.

\section{Implications for Accretion Models and Young Brown Dwarfs}

The combination of SDSS and 2MASS photometry can be used to provide constraints on accretion luminosity, the inner radius of the circumstellar disk, and the magnetic field and rotational velocity of the young object. The intrinsic $u$ band excess can be used to infer an accretion luminosity in that band, and hence a lower limit on the total accretion luminosity. Gullbring et al. (1988) find a well constrained relation between the shock emission in the Johnson $U$ band and the total accretion luminosity.

The magnetospheric accretion model (Königl 1991) allows prediction of the mass accretion rate from the truncation radius and the accretion luminosity given the stellar mass and radius. This in turn places constraints on the strength of the stellar magnetic dipole and the stellar rotational velocity given that the disk truncates at the co-rotational radius.

For objects that exhibit a circumstellar disk signature, we can ask: 1) How do conditions vary in the inner circumstellar disk as a function of mass? 2) Is there a trend in the $\mathrm{H}-\mathrm{K}$ excess consistent with purely a decrease in contrast against the stellar photosphere? 3) How does the mass accretion rate vary with mass and age? 4) Is there a dependence of the magnetic field strength on mass for objects of a similar age? 5) Do we see indications that the magnetic field decays with time as expected if it is primarily inherited from the parent molecular cloud?

\section{Program Status}

Examination of the SDSS data set is pending reprocessing of the "Orion" scans using the current version of the photometric pipeline.

Acknowledgments. Funding for the creation and distribution of the SDSS Archive has been provided by the Alfred P. Sloan Foundation, the Participating Institutions, the National Aeronautics and Space Administration, the National Science Foundation, the U.S. Department of Energy, the Japanese Monbukagakusho, and the Max Planck Society.

The SDSS Web site is http://www.sdss.org/.

\section{References}

Baraffe, I. et al. 1998, A\&A, 337, 408

Gullbring, E. et al. 1998, ApJ, 492, 323

Königl, A. 1991, ApJ, 370, L39

Rebull, L. M. et al. 2002, AJ, 125, 1528

Smith, J. A. et al. 2002, AJ, 123, 2121

York, D. G. et al. 2000, AJ, 120, 1579 